ARTICLE

Received 5 May 2015 | Accepted 11 Aug 2015 | Published 21 Sep 2015

DOI: $10.1038 /$ ncomms 9341

OPEN

\title{
Two classes of regulatory subunits coassemble in the same BK channel and independently regulate gating
}

Vivian Gonzalez-Perez ${ }^{1}$ Xiao-Ming Xia ${ }^{1} \&$ Christopher J. Lingle ${ }^{1}$

High resolution proteomics increasingly reveals that most native ion channels are assembled in macromolecular complexes. However, whether different partners have additive or cooperative functional effects, or whether some combinations of proteins may preclude assembly of others are largely unexplored topics. The large conductance $\mathrm{Ca}^{2+}$-and-voltage activated potassium channel (BK) is well-suited to discern nuanced differences in regulation arising from combinations of subunits. Here we examine whether assembly of two different classes of regulatory proteins, $\beta$ and $\gamma$, in BK channels is exclusive or independent. Our results show that both $\gamma 1$ and up to four $\beta 2$-subunits can coexist in the same functional BK complex, with the gating shift caused by $\beta 2$-subunits largely additive with that produced by the $\gamma 1$-subunit(s). The multiplicity of $\beta: \gamma$ combinations that can participate in a BK complex therefore allow a range of BK channels with distinct functional properties tuned by the specific stoichiometry of the contributing subunits.

\footnotetext{
${ }^{1}$ Department of Anesthesiology, Washington University School of Medicine, St. Louis, Missouri 63110, USA. Correspondence and requests for materials should be addressed to C.J.L. (email: clingle@morpheus.wustl.edu).
} 
P roteomic $^{1,2}$ and functional ${ }^{2-6}$ studies have revealed many partners that interact with BK channels, some of which are known to confer distinct tissue-specific functional properties on the $\mathrm{BK}$ pore-forming subunit. $\mathrm{BK}$ channels are dually activated by membrane depolarization and increases in intracellular $\left[\mathrm{Ca}^{2+}\right]$ (refs 7-9). The minimal functional unit of a BK channel is a homotetramer of pore-forming $\alpha$-subunits (Fig. 1a), each containing intrinsic voltage-sensing and $\mathrm{Ca}^{2+}$. sensing domains. However, BK channels may also contain any of two different families of regulatory proteins, $\beta$ and $\gamma$, which help define tissue-specific functional properties of a BK complex. The four members of the $\beta$ family ( $\beta 1-\beta 4)$ and the four members of the recently discovered $\gamma$ family $(\gamma 1-\gamma 4)$ differentially regulate BK function, influencing $\mathrm{Ca}^{2+}$-dependence of activation ${ }^{2,5,6,10,11}$, current inactivation ${ }^{4,5,12,13}$ and even pharmacology ${ }^{5,14}$.

$\beta$ - and $\gamma$-subunits are unrelated proteins with very different predicted structural topology. $\beta$-subunits contain two transmembrane (TM) segments linked by an extracellular loop bridged by multiple disulfide linkages and intracellular $\mathrm{C}$ and $\mathrm{N}$ termini ${ }^{15}$; $\gamma$-subunits contain a single TM segment, a cytosolic C terminus, and an extracellular $\mathrm{N}$ terminus with a large leucine-rich repeatcontaining motif ${ }^{6,16}$ (Fig. 1b). Specific structural information for $\beta$ - and $\gamma$-subunits is not available. For $\beta$-subunits, potential positions of TM segments in relation to $\alpha$-subunit TM segments have been proposed ${ }^{17-19}$ (Fig. 1c). Individual BK channels can contain 0-4 $\beta$-subunits, with each subunit contributing in an

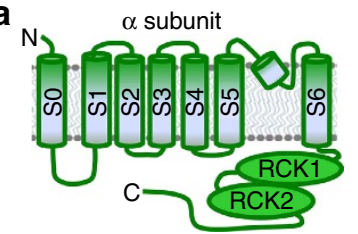

b

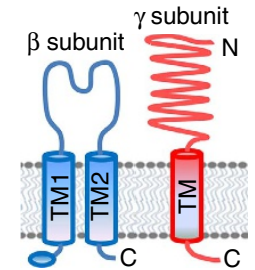

C

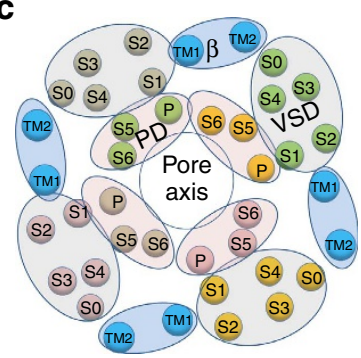

h

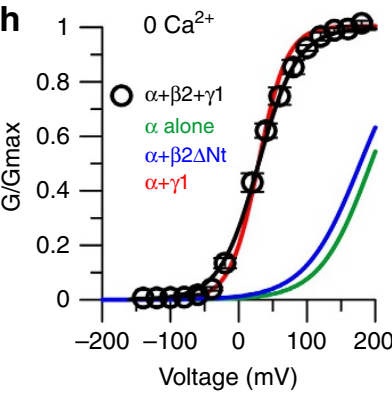

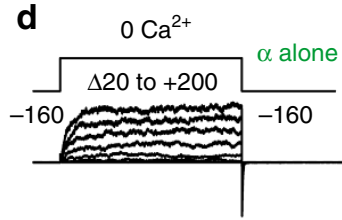

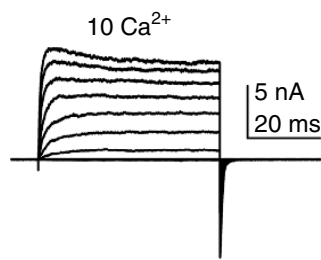

e
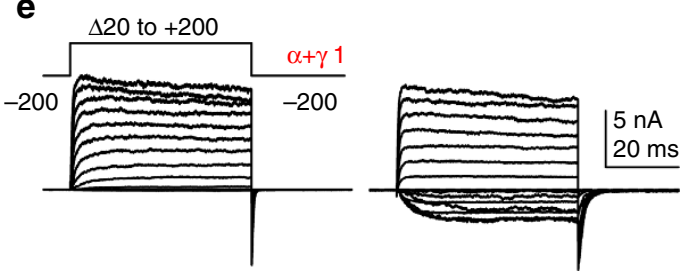

f
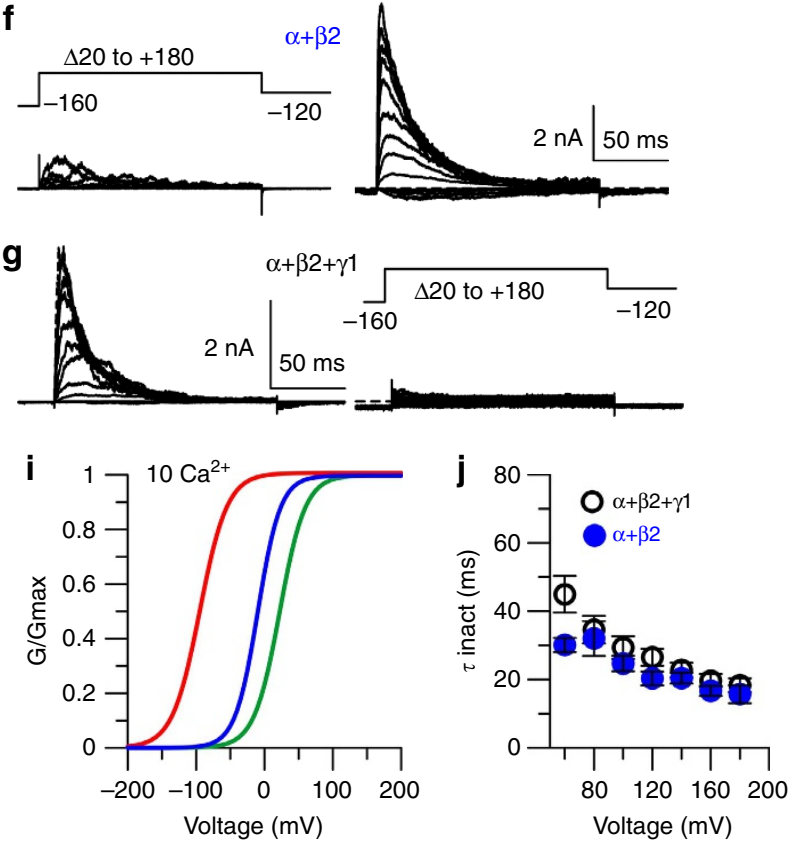

Figure 1 | Coexpression of $\boldsymbol{\alpha}+\boldsymbol{\beta 2}+\gamma \mathbf{1}$ subunits results in all BK channels containing both $\boldsymbol{\beta}$ - and $\boldsymbol{\gamma}$-subunits. (a-b) Cartoons of the predicted topology of $\alpha-, \beta 2-$ and $\gamma 1$-subunits. The basic topology of $\beta 2$ - and $\gamma 1$-subunits are shared with other members of their respective subunit families. (c) Schematic of deduced position of $\alpha$ - and $\beta 1$-subunit TM segments in a BK channel complex viewed at the extracellular face ${ }^{17}$. Grey, pink and blue ellipses highlight voltage sensor domain (VSD), pore domain (PD) and $\beta$-subunit TMs, respectively. ( $\mathbf{d}-\mathbf{g}$ ) Typical currents arising from (co)expression of BK subunits in oocytes: $\alpha$ alone, $\alpha+\gamma 1, \alpha+\beta 2$, or $\alpha+\beta 2+\gamma 1$, respectively, at 0 (left) or $10 \mu \mathrm{M}$ intracellular [Ca $\left.{ }^{2+}\right]$ (right); left and right currents were from the same patch using the same voltage protocol (depicted for each case). (h-i) Comparison among activation curves (G-V) at $0 \mathrm{Ca}^{2+}$ or $10 \mu \mathrm{M}$ of $\mathrm{Ca}{ }^{2+}$, respectively, generated from sets of experiments as in panels $(\mathbf{d}-\mathbf{g})$. Notice the overlap between activation curves at $0 \mathrm{Ca}^{2}+$ of $\alpha+\gamma 1$ and $\alpha+\beta 2+\gamma 1$. G-V fits to single Boltzmann distributions yielded the following parameters: At $0 \mathrm{Ca}^{2+}, \alpha$-alone $\left(V_{\mathrm{h}}=193 \pm 6 \mathrm{mV}, z=0.7 \pm 0.02 \mathrm{e}_{0}, n=5\right) ; \alpha+\beta 2 \Delta \mathrm{Nt}$ $\left(V_{\mathrm{h}}=182 \pm 4 \mathrm{mV}, z=0.7 \pm 0.03 \mathrm{e}_{0}, n=5\right) ; \alpha+\gamma 1\left(V_{\mathrm{h}}=29.0 \pm 4.7 \mathrm{mV}, z=1.3 \pm 0.08 e_{0}, n=7\right) ; \alpha+\beta 2+\gamma 1\left(V_{\mathrm{h}}=28.9 \pm 3.4 \mathrm{mV}, z=0.9 \pm 0.04 e_{0}\right.$ $n=9)$. At $10 \mu \mathrm{M}$ of $\mathrm{Ca}^{2+}, \alpha$-alone $\left(V_{\mathrm{h}}=22.6 \pm 3 \mathrm{mV}, z=1.4 \pm 0.12 \mathrm{e}_{0}, n=5\right) ; \alpha+\beta 2 \Delta \mathrm{Nt}\left(V_{\mathrm{h}}=-9.2 \pm 4.4 \mathrm{mV}, z=1.6 \pm 0.87 \mathrm{e}_{0}, n=5\right) ; \alpha+\gamma 1$ $\left(V_{h}=-95.1 \pm 3.9 \mathrm{mV}, z=1.4 \pm 0.05 e_{0}, n=8\right)$. (j) Averages of $\tau_{\text {inact }}$ at voltages up to +180 for $\alpha+\beta 2+\gamma 1$ at $0 \mathrm{Ca}^{2+}(n=9)$ and $\alpha+\beta 2$ at $10 \mathrm{Ca}^{2+}$ $(n=6)$, respectively. All errors are s.e.m. 
energetically independent fashion to shift $\mathrm{BK}$ gating ${ }^{20}$. For $\gamma$-subunits, both the position in the channel complex and the $\alpha: \gamma$ stoichiometry remain unknown. In heterologously expressed $\alpha+\gamma 1$ channels, the $\gamma 1$-induced gating shift occurs in an all-or-none manner, consistent with an elementary functional unit of $\gamma 1$ (for example, monomer, dimer and tetramer) being sufficient to produce the full effect ${ }^{21}$. Here to determine whether different types of regulatory subunits coassemble in the same BK channels, we take advantage of the distinctive functional properties conferred on BK channels by $\beta 2$ - and $\gamma 1$-subunits. Using ensemble and single molecule approaches we report that $\gamma 1$ and 1-4 $\beta 2$-subunits can be simultaneously present in the same $\mathrm{BK}$ channel and independently contribute to modulation of BK function.

\section{Results}

B2- and $\gamma 1$-subunits coassemble in the same BK channel. The $\gamma 1$-subunit produces a remarkable negative shift of $120-140 \mathrm{mV}$ in the voltage-range of $\mathrm{BK}$ channel activation either in the presence or absence of $\mathrm{Ca}^{2+(2)}$ (Fig. 1d,e,h,i). While BK channels composed of $\alpha$ alone require at least $10 \mu \mathrm{M} \mathrm{Ca}^{2+}$ to show appreciable open probability over physiologically relevant voltages, (Fig. $1 \mathrm{~h}, \mathrm{i}), \alpha+\gamma 1$ channels show a similar probability in the total absence of intracellular $\mathrm{Ca}^{2+}$ (Fig. 1e,h). The ability of $\gamma 1$ to shift $\mathrm{BK}$ gating in $0 \mathrm{Ca}^{2+}$ contrasts with the absence of a gating shift produced by $\beta 2$ (Fig. 1h) under the same conditions ${ }^{22}$.

The most readily identifiable effect of the $\beta 2$-subunit is essentially complete inactivation that occurs following channel activation (Fig. 1f). Inactivation arises from the cytosolic $\mathrm{N}$ terminus of the $\beta 2$-subunit ${ }^{4,5,23}$. Since BK channels can contain 1-4 $\beta 2$-subunits, each acting independently, single $\alpha+\beta 2$ channels exhibit one of four distinct inactivation rates ${ }^{20}$. The inactivation time constant $\left(\tau_{\text {inact }}\right)$ therefore provides a measure of $\beta 2$-subunit stoichioimetry within either a channel population or individual channels ${ }^{20}$. Although $\beta 2$ produces little gating shift at $0 \mathrm{Ca}^{2+(23)}$ (Fig. 1h), gating of $\alpha+\beta 2$ channels at $10 \mu \mathrm{M} \mathrm{Ca}^{2+}$ is shifted about $40 \mathrm{mV}$ leftward compared with $\alpha$ channels $\mathrm{s}^{3,5,20}$ which is more clearly observed using a non-inactivating $\beta 2$ variant $(\beta 2 \Delta \mathrm{Nt})$ (Fig. 1i). Thus, for $\alpha+\beta 2$ channels, voltage steps up to $+180 \mathrm{mV}$ only weakly activate $\mathrm{BK}$ currents at $0 \mathrm{Ca}^{2+}$ (Fig. 1f, left), while $10 \mu \mathrm{M} \mathrm{Ca}^{2}+$ produces robust activation of an inactivating current (Fig. 1f, right), whose $\tau_{\text {inact }}$ approaches a limiting value of $\sim 20 \mathrm{~ms}$ (Fig. 1j), indicating an average of 4 $\beta 2$-subunits/channel in the population ${ }^{20}$. Another physiologically relevant property of inactivating channels, the voltage dependence of steady-state inactivation (SS-inactivation), is also an indicator of the presence of $\beta 2$-subunits. During exposure of $\alpha+\beta 2$ channels to $10 \mu \mathrm{M} \mathrm{Ca}{ }^{2+}$, the availability of noninactivated channels to open is very low at voltages above $-50 \mathrm{mV}$ (Fig. 2a,c) with half-channel availability $\left(V_{\mathrm{h}}\right)$ at about $-110 \mathrm{mV}$. Together, the distinctive effects of $\beta 2$ - and $\gamma 1$-subunits provide useful signatures to verify the presence of $\beta 2$ and $\gamma 1$ in BK channels resulting from the coexpression of $\alpha+\beta 2+\gamma 1$.

We therefore coexpressed $\gamma 1+\beta 2$ subunits with $\alpha$ at relative mole fractions of message that would produce full effects of either $\gamma 1$ or $\beta 2$ alone $^{20,21}$. The coexpression of $\alpha+\beta 2+\gamma 1$ subunits results in a prominent inactivating outward current even with 0 $\mathrm{Ca}^{2+}$ (Fig. 1g, left), similar to currents resulting from the expression of $\alpha+\beta 2$ subunits when activated by $10 \mu \mathrm{M} \mathrm{Ca}{ }^{2+}$ (Fig. 1f). The complete inactivation of currents obtained after coexpression of $\alpha+\beta 2+\gamma 1$ subunits indicates that essentially all channels contain $\beta 2$-subunits. Furthermore, that in such patches the voltage dependence of activation at $0 \mathrm{Ca}^{2+}$ is shifted more
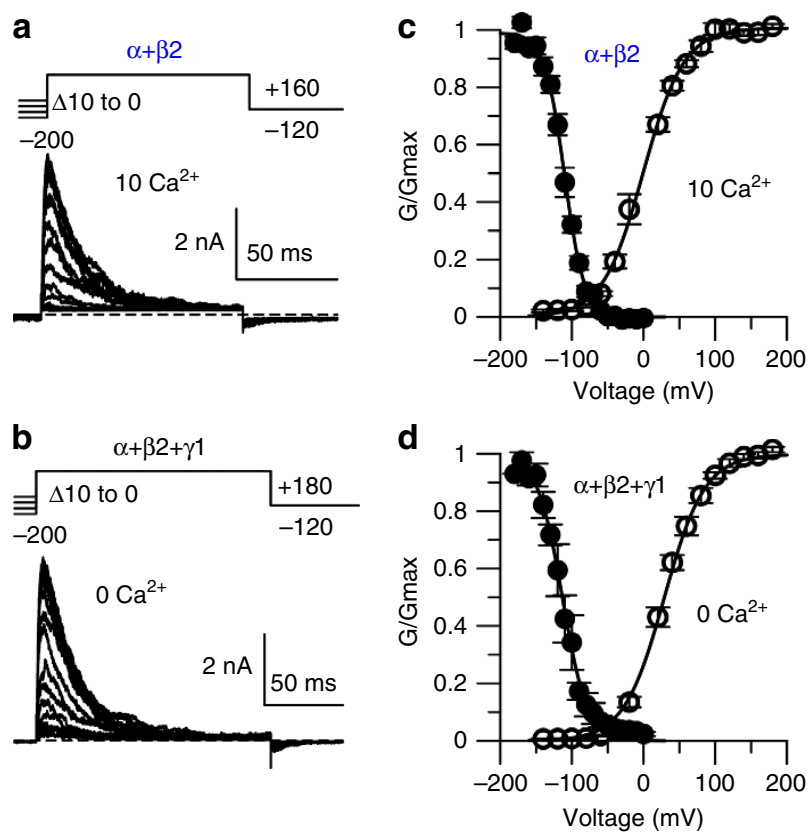

Figure 2 | The voltage dependence of the fraction of channels available to activate also reflects the coassembly of $\beta 2$ - and $\gamma 1$-subunits in the same channels. (a-b) Examples of the fractional availability of channels as function of the conditioning potential from oocytes injected with $\alpha+\beta 2$ $\left(10 \mu \mathrm{M} \mathrm{Ca}^{2+}\right)$ or $\alpha+\beta 2+\gamma 1\left(0 \mathrm{Ca}^{2+}\right)$, respectively. Currents were evoked by the indicated protocols using conditioning potentials of $100 \mathrm{~ms}$ duration. (c-d) Curves of activation (open symbols) and fractional availability (filled symbols) as function of voltage obtained at $10 \mu \mathrm{M}$ of $\mathrm{Ca}^{2}+$ for $\alpha+\beta 2$ or $0 \mathrm{Ca}^{2+}$ for $\alpha+\beta 2+\gamma 1$ channels, respectively, are plotted together. $G-V$ fits to single Boltzmann distributions yielded the following parameters: $\alpha+\beta 2$ SS-inactivation $\left(V_{h}=-110.8 \pm 2.3 \mathrm{mV}\right.$, $\left.z=1.81 \pm 0.11 e_{0}, n=7\right) \alpha+\beta 2$-activation $\left(V_{h}=-6.7 \pm 2.4 \mathrm{mV}\right.$, $\left.z=0.96 \pm 0.31 e_{0}, n=6\right) ; \alpha+\beta 2+\gamma 1$-SS-inactivation $\left(V_{h}=-113.5 \pm 5.3 \mathrm{mV}\right.$, $\left.z=1.56 \pm 0.12 e_{0}, n=4\right), \alpha+\beta 2+\gamma 1$-activation (also shown in Fig. 1h). All errors are s.e.m.

than $-120 \mathrm{mV}$ (Fig. 1h) is diagnostic for the presence of $\gamma 1$. When the same patch is activated with $10 \mu \mathrm{M} \mathrm{Ca}^{2+}$, essentially no current is observed (Fig. 1g, right) which suggests that $\alpha+\beta 2+\gamma 1$ channels are constitutively inactivated with $10 \mu \mathrm{M}$ $\mathrm{Ca}^{2}+$ at $-160 \mathrm{mV}$. Indeed, the fractional availability of $\alpha+\beta 2+\gamma 1$ currents at $0 \mathrm{Ca}^{2}+$ (Fig. 2b) exhibits a voltage dependence very similar to that observed with $10 \mu \mathrm{M} \mathrm{Ca}^{2+}$ for $\alpha+\beta 2$ currents (Fig. $2 c-d$ ). The markedly leftward-shifted steady-state inactivation curve of $\alpha+\beta 2+\gamma 1$ channels also confirms that both $\beta 2$ - and $\gamma 1$-subunits can coassemble in the same BK channels.

$\beta 2$ and $\gamma 1$ occupy distinct positions in the BK channel complex. We next wondered whether channels containing both $\beta 2+\gamma 1$ subunits can contain a full set of four $\beta 2$-subunits. We imagined two kinds of assembly scenarios: (1) an independent model (Fig. 3a), where the presence of $\gamma 1$-subunits does not hinder the ability of four $\beta 2$-subunits to fully populate a BK channel ( $\beta 2$ and $\gamma 1$ occupy different positions), or (2) an occlusive model (Fig. 3b), where the presence of $\gamma 1$ excludes the assembly of $\beta 2$-subunits ( $\beta 2$ and $\gamma 1$ occupy overlapping positions). These two models can be tested by examination of the $\tau_{\text {inact }}$ arising from a set of single $\alpha+\beta 2+\gamma 1$ channels obtained under conditions in which relative $\beta 2$ subunit expression varies ${ }^{20}$. If the independent model is valid, $\gamma 1$-containing inactivating single channels should exhibit four distinct $\tau_{\text {inact }}$ (ref. 20), while the finding of less than four 


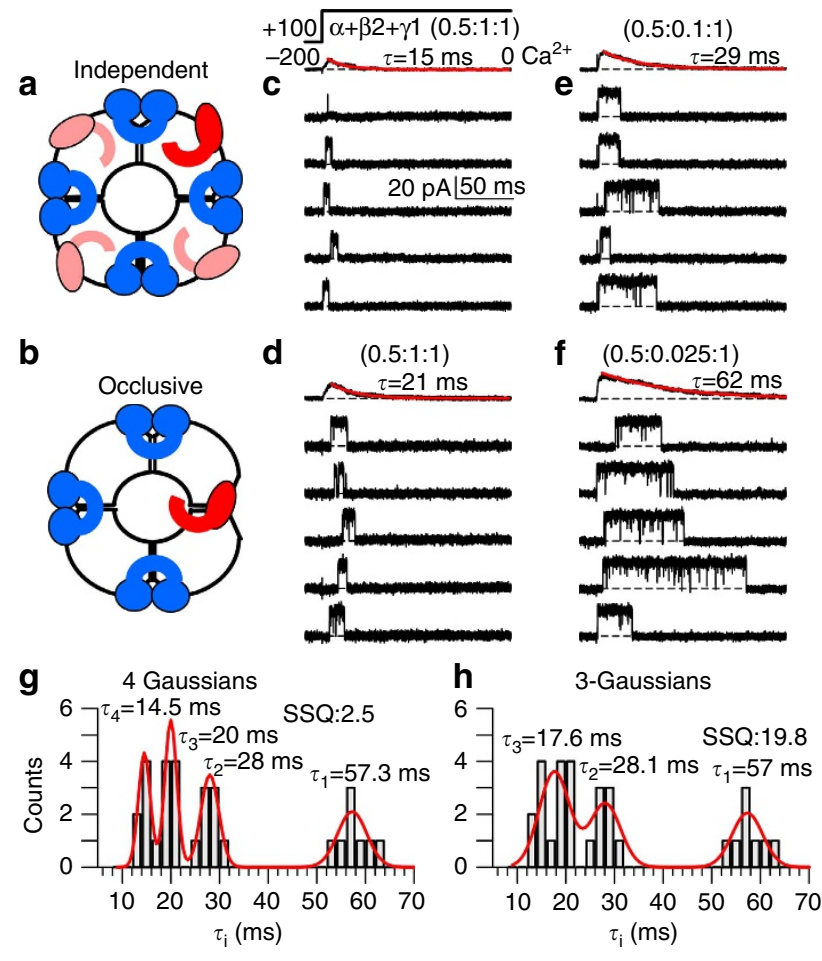

Figure 3 | Single $\alpha+\beta 2+\gamma 1$ channels simultaneously exhibit the maximal $\boldsymbol{\gamma} \mathbf{1}$-induced effect and up to four $\boldsymbol{\beta 2}$-subunits. (a-b) Cartoons representing two distinctive assembly models: where $\alpha$, white; $\beta 2$, blue; $\gamma 1$, red. (c-f) Representative single channels obtained from various $\alpha: \beta 2: \gamma 1$ injection ratios (given above each set of traces). For each example, five consecutive traces of current recorded at $0 \mathrm{Ca}^{2}+$ are shown together with the ensemble current average from 100 total traces recorded in the same conditions. Red lines: fit of a single exponential with the indicated $\tau_{\text {inact }}$ (g-h) Grey bars represent the distribution of $\tau_{\text {inact }}$ from 31 single-channels (bin size: $2 \mathrm{~ms}$ ). Red lines represent fits of the binned data to four- or three-component Gaussian distributions, respectively, both with the s.d. $\left(s_{n}\right)$ constrained such that $s_{1 \geq} s_{2 \geq} s_{3} \ldots s_{n}$. Mean values $\left(\tau_{n}\right)$ and sum of the squares of the errors (SSQ) obtained from each fit are indicated. The other parameters of each Gaussian component, amplitude $\left(A_{n}\right)$ and standard deviation, of the distributions are: four-component distribution $\left(A_{1}=2.1\right.$, $\left.s_{1}=3.0 ; A_{2}=3.5, s_{2}=1.8 ; A_{3}=5.6, s_{3}=1.2 ; A_{4}=4.3, s_{4}=1.2\right)$ and threecomponent distribution $\left(A_{1}=2.05, s_{1}=3.1 ; A_{2}=2.4, s_{2}=3.1 ; A_{3}=3.6\right.$, $\left.s_{3}=3.1\right)$.

inactivation behaviours supports the occlusive model. By keeping both $\alpha$ and $\gamma 1$ constant but decreasing $\beta 2$ in the RNA injection mix, we obtained a set of single channels that exhibit inactivation consistent with the presence of $\beta 2$, but gating shifts consistent with the presence of $\gamma 1$ (Supplementary Fig. 1). The $\tau_{\text {inact }}$ observed from 31 channels appeared to group into four behaviours (Fig. 3c-f). For channels that might potentially contain either three or four $\beta 2$-subunits, the expected differences in mean inactivation time constant are not great. We therefore examined in detail the ability of three- and fourcomponent Gaussian functions to fit the distribution. In evaluating fits of the distribution, we considered the impact of two criteria. First, since inactivation arises from independent inactivation domains ${ }^{20}$, the faster components should bear a simple arithmetic relationship to the slowest component. Second, the s.d. for any component, although perhaps not wellconstrained by the data, should be smaller than for any slower components, such that $s_{1} \geq s_{2} \geq s_{3} \geq s_{4}$. Various considerations in the fitting process are given in the Methods and in Supplementary Fig. 2. For the case that the s.d. are constrained as mentioned or
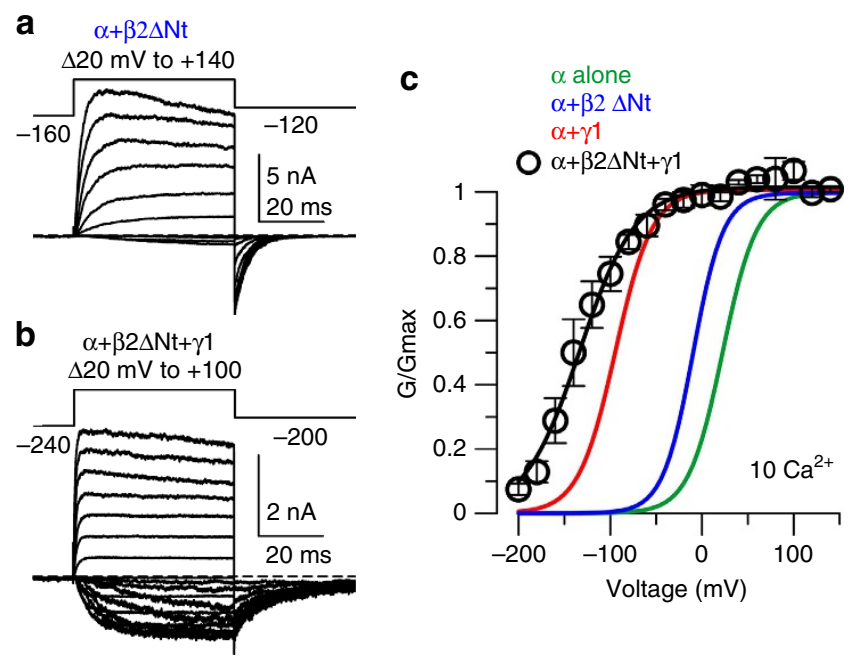

Figure $4 \mid \gamma \mathbf{1}$ and $\beta 2$ induced gating shifts are largely additive.

(a-b) Currents arising from coexpression of $\alpha+\beta 2$-inactivation removed $(\beta 2 \Delta \mathrm{Nt})$ (a) or $\alpha+\beta 2 \Delta \mathrm{Nt}+\gamma 1$ (b) with $10 \mu \mathrm{M} \mathrm{Ca}^{2+}$. (c) Comparison of the gating shift obtained by $\beta 2$ with or without the $\gamma 1$ expression. $\beta 2$ produces an approximately $30-35 \mathrm{mV}$ shift with or without $\gamma 1$. With $10 \mu \mathrm{M}$ $\mathrm{Ca}^{2}+, V_{\mathrm{h}}$ for $(\alpha$ alone $)=+22.6 \pm 3.4 \mathrm{mV},(\alpha+\gamma 1)=-95.1 \pm 3.9 \mathrm{mV}$, $(\alpha+\beta 2 \Delta \mathrm{Nt})=-9.2 \pm 4.4 \mathrm{mV}$ and $(\alpha+\beta 2 \Delta \mathrm{Nt}+\gamma 1)=-134.7 \pm 8.8 \mathrm{mV}$ $(n=5) ; \Delta V(\beta 2)=31.8, \Delta V(\gamma 1)=117.7, \Delta V(\beta 2 \Delta N \mathrm{Nt}+\gamma 1)=157.3$. All errors are s.e.m.

where the mean values of each component are defined by the slowest component, the $\tau_{\text {inact }}$ distribution is better fit by a fourcomponent than by a three-component Gaussian distribution (Fig. 3g,h, Supplementary Fig. 2), indicating that channels containing the $\gamma 1$-induced effect can also contain one, two, three or four $\beta 2$-subunits. In the case of the three-component Gaussian, if the constraint on s.d. is relaxed, the best fit is reached with the fastest component having the largest s.d. of any component, suggestive that this fast component in fact arises from two populations. The superior fit of the four-component Gaussian supports the existence of individual channels containing $4 \beta 2$ and at least one $\gamma 1$-subunit indicating that both types of auxiliary subunits occupy independent positions in the channel complex (Fig. 3a).

$\beta 2$ and $\gamma 1$ independently contribute to BK gating shifts. Both $\beta 2$ and $\gamma 1$ produce leftward shifts in BK gating, but their effects are likely mediated by different mechanisms. Whereas the $\gamma 1$-effect can be explained by stronger coupling between the voltage-sensor movement and channel activation ${ }^{2}$, the effects of $\beta 2$ appear more complex $22,24-26$. We asked whether $\gamma 1$ and $\beta 2$ effects might be additive or occlusive. For better elucidation of the $\beta 2$ effects, we compared the gating shift resulting from the coexpression of $\gamma 1+\beta 2 \Delta \mathrm{Nt}$ versus that produced by each construct separately when coexpressed with BK- $\alpha$-subunits (Fig. 4). The $V_{\mathrm{h}}$ arising from the simultaneous presence of $\gamma 1$ and $\beta 2 \Delta \mathrm{Nt}$ approximately reflects the sum of the independent effects of $\gamma 1$ and $\beta 2 \Delta \mathrm{Nt}$ alone. These results indicate that, whatever the underlying molecular mechanism of the $V_{\mathrm{h}}$ shift produced by the $\gamma 1$-subunit, it is predominantly energetically independent of that produced by the $\beta 2$-subunit. Furthermore, there is no inhibitory allosteric coupling between the auxiliary subunits themselves.

Other $\beta$-subunits also coassemble with $\gamma 1$ in BK channels. Can other $\beta$-subunits also coassemble with $\gamma 1$ in BK channels? 
An earlier report suggested that the presence of $\beta 1$-subunits may occlude the ability of $\gamma 1$ to produce its gating shift ${ }^{2}$. Since the overexpression of one subunit might influence the successful expression of another, we used proportions of RNA for each subunit similar to those used in testing $\gamma 1+\beta 2$ coassembly with $\alpha$-subunits. Using a $\beta 1$ construct in which its $\mathrm{N}$ terminus was replaced by the $\beta 2-\mathrm{N}$ terminus $(\beta 1 / \beta 2 \mathrm{Nt})$ so that inactivation reports the presence of $\beta 1$, we found that all BK channels resulting from coexpression of $\alpha+\beta 1 / \beta 2 \mathrm{Nt}+\gamma 1$ simultaneously contain both types of regulatory subunits (Supplementary Fig. 3).

\section{Discussion}

The present work unambiguously shows that two different types of non-pore-forming regulatory subunits, $\beta 2$ and $\gamma 1$, can coassemble in the same functional BK channel and independently regulate channel function. The $\alpha+\beta 2+\gamma 1$ combination generates a BK channel with novel functional properties, which subtly change depending on the stoichiometry of $\beta 2$ in the multimeric complex. In a normal cellular environment, the simultaneous presence of $\beta 2+\gamma 1$ in $\mathrm{BK}$ channels might effectively remove them from availability for activation especially when intracellular calcium is increased. However, at $0 \mathrm{Ca}^{2+}$, such channels would not be fully inactivated (Fig. 2d): the window of overlap between the fractional availability curve and the activation curve spans approximately -60 to $-30 \mathrm{mV}$, a range corresponding to normal resting potentials in many types of cells, including neurons. This essentially defines a range of voltage over which any contributions of $\alpha+\beta 2+\gamma 1$ channels might be dynamically regulated by inactivation. Given the large conductance of the BK channel, only a small fraction of the total BK population would have to undergo cycles of inactivation, recovery from inactivation and activation to have some influence on cell excitability. Although it is unknown whether $\beta 2+\gamma 1$ subunits are simultaneously present in any cell-type, reported message levels of both $\beta 2$ and $\gamma 1$-subunits in some tissues ${ }^{11,27}$ support the possibility that $\beta$ - and $\gamma$-subunits will copartner in at least some cells. For any cells which may express both a $\beta 2$ - and a $\gamma 1$-subunit, our results establish that these two distinct regulatory partners of BK channels can simultaneously and independently contribute to modulation of BK function and do not appear to hinder the assemble of each other into a channel. The possibility of coassembly would also likely apply to other members of the $\beta$ and $\gamma$ families, as supported by our results with $\beta 1+\gamma 1$ subunits. Our findings highlight not only the critical importance of defining the identity of protein partners in native multimeric complexes within a cell or a specific cell location, but also the importance of understanding how individual contributions of distinct regulatory components and their stoichiometry can define fundamental properties of the complex.

\section{Methods}

Constructs. Primary constructs were mouse $\alpha$ (SLO1) (ref. 9), human LRRC26 $(\gamma 1)$ (ref. 11) and human $\beta 2$ (ref. 23). In some experiments $\beta 2 \Delta \mathrm{Nt}$ (the first 33 amino acids were removed from the $\beta 2-\mathrm{N}$-terminal) or $\beta 1 / \beta 2 \Delta \mathrm{Nt}$ (first 43 amino acids from the $\beta 2-\mathrm{N}$ terminus replaced the first 11 amino acids of the $\beta 1-\mathrm{N}$ terminus) were used.

Oocyte expression. Stage IV Xenopus laevis oocytes were used for channel expression. The complementary RNAs (cRNAs) of all constructs were prepared at approximately $1 \mu \mathrm{g}^{-1}$. For macroscopic recordings, cRNA mixes containing (molar ratio): mSLO1 alone, mSLO1 + h $\beta 2$ (1:6.5), mSLO1 + hLRRC26 (1:4) or $\mathrm{mSLO} 1+\mathrm{h} \beta 2+$ hLRRC26 (1:6.5:4), were diluted 1:5 before injection. For single channel recordings, mSLO1:h $\beta 2:$ hLRRC26 (1: 0.65:4) and (1:0.16:4) were diluted $1: 20-1: 100$ before injection. $\beta 2 \Delta \mathrm{Nt}$ or $\beta 1 / \beta 2 \Delta \mathrm{Nt}$ cRNAs were used at the same molar ratio as $\beta 2$ in some experiments. Oocytes were used $2-5$ days after injection, except for single channel recordings in which they were used 1-2 days after injection. Maintenance of frogs and isolation of oocytes following procedures approved by the Washington University in St Louis Institutional Animal Care and Use Committee.

Electrophysiology. Borosilicate glass capillaries (1B150F-4, World Precision Instruments) were pulled to diameters resulting in resistances of 1-2 or 5-6 $\mathrm{M} \Omega$ for macroscopic and single channel recordings, respectively. Pipettes were coated with Sylgard 184 (Dow Chemical Corp.) and fire-polished. Currents were recorded in the inside-out patch configuration using an Axopatch 200B amplifier (Molecular Devices) and the Clampex program from the pClamp software package (Molecular Devices). Gigaohm seals were formed in frog Ringer (in mM, $115 \mathrm{NaCl}, 2.5 \mathrm{KCl}$ 1.8 $\mathrm{CaCl}_{2}, 10 \mathrm{HEPES}, \mathrm{pH} 7.4$ ) and, after patch excision, moved into flowing test solutions. The pipette/extracellular solution was (in $\mathrm{mM}$ ): $140 \mathrm{~K}$-methanesulfonate, $20 \mathrm{KOH}, 10 \mathrm{HEPES}, 2 \mathrm{MgCl}_{2}$, pH 7.0. Test solutions of different $\mathrm{Ca}^{2+}$ contained $^{28}$ $140 \mathrm{mM}$ methanesulfonate, $20 \mathrm{mM} \mathrm{KOH}, 10 \mathrm{mM}$ HEPES with $\mathrm{pH}$ adjusted to 7.0 (ref. 28). N-(2-hydroxyethyl)ethylenediamine-triacetic acid (HEDTA) was used for

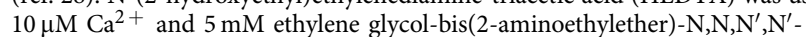
tetraacetic acid (EGTA) for $0 \mu \mathrm{M} \mathrm{Ca}^{2+}$ solutions. The $10 \mu \mathrm{M} \mathrm{Ca}^{2+}$ solution was titrated to appropriate $\mathrm{pCa}$ with Ca-MES and calibrated against solutions of defined $\mathrm{Ca}^{2+}$ concentrations (World Precision Instruments) using a $\mathrm{Ca}^{2+}$ sensitive electrode. Test solutions were applied directly to patches via a large bore pipette tip containing multiple independent solution lines. All experiments were at room temperature $\left(\sim 22-25^{\circ} \mathrm{C}\right)$.

Data analysis. Analysis were made using Clampfit (Molecular Devices). For noninactivating currents, conductance values $(G)$ were obtained from the tail currents, while for inactivating currents the peak current was used. $G-V$ data sets were fitted with a single Boltzmann function: $G(V)=\frac{G \max }{1+\frac{z F\left(V-V_{h}\right)}{R T}}$, where $V_{\mathrm{h}}$ represents the

voltage of half activation and $z$ is the valence of the voltage dependence. Notice that the $\alpha+\gamma 1$ injection ratio used (1:4 molar ratio) results in around $90 \%$ of BK channels expressed containing the full $\gamma 1$-induced effect ${ }^{21}$, in which case the fit to a single Boltzmann distribution provides a good estimation of $V_{\mathrm{h}}$ and $z$ for $\alpha+\gamma 1$ currents. Macroscopic inactivation time constants $\left(\tau_{\text {inact }}\right)$ were obtained by fitting each current decay to a single exponential function.

All error estimates are SEM.

Single-channel traces were first processed using digital subtraction of leak and capacitive current defined from traces lacking any channel opening. $\tau_{\text {inact }}$ for each single channel was estimated from the ensemble current average of 50-100 identical sweeps recorded at $0 \mathrm{Ca}^{2+}$. The histogram of $\tau_{\text {inact }}$ was generated using a bin size of $2 \mathrm{~ms}$ and the distribution was fitted to the sum of three or four components Gaussian function:

$$
f(x)=\sum_{n=1}^{i} A_{n} e^{\frac{\left(x-t_{n}\right)^{2}}{2 s_{n}^{2}}}
$$

where $A_{n}, \tau_{n}$ and $s_{n}$ represent the amplitude, mean- $\tau_{\text {inact }}$ and s.d. of each component, respectively, with $n=1$ for the parameters of the slowest inactivating component, $n=2$ for the second slower and so on. Since each measured $\tau_{\text {inact }}$ represents the mean of an exponentially distributed population, the s.d. for the average of those grouped in the slowest component of the $\tau_{\text {inact-distribution should }}$ be larger than for faster components, such that $s_{1 \geq} s_{2} s_{3 \geq} s_{4}$. Fitting to the sum of three or four Gaussian functions was first made allowing free variation of all fitting parameters. However, in both cases the rank of s.d. do not follow the expected criteria mentioned before: the four-component fit yields a very narrow thirdcomponent (very small $s_{3}$ ), while the three-component fit results with the fastest component having the largest s.d. $\left(s_{3}\right)$ among all components (Supplementary Fig. $2 \mathrm{a}-\mathrm{b})$. Two factors may contribute to such deviations: first, that the numbers of entries in the histogram are insufficient to accurate define all aspects of each component and, second, that some components may actually arise from multiple components. We then constrained the s.d. in both cases to fulfil the expected criteria $\left(s_{1 \geq} s_{2} \geq \ldots s_{n}\right)$ (see results in Fig. $3 g-h$ ). Furthermore, we took advantage of the idea that inactivation results from the independent movement of each inactivation domain to the central cavity of the pore ${ }^{20}$. Based on this latter consideration, in some cases we also constrained all mean values of each component to be dependent on the slowest component (Supplementary Fig. 2c-d). All cases yield better fits using four than three-component Gaussian distributions.

Chemicals. Salts and buffers were obtained from Sigma.

\section{References}

1. Berkefeld, H. et al. BKCa-Cav channel complexes mediate rapid and localized $\mathrm{Ca}^{2+}$-activated $\mathrm{K}^{+}$signaling. Science 314, 615-620 (2006).

2. Yan, J. \& Aldrich, R. W. LRRC26 auxiliary protein allows BK channel activation at resting voltage without calcium. Nature 466, 513-516 (2010).

3. Brenner, R., Jegla, T. J., Wickenden, A., Liu, Y. \& Aldrich, R. W. Cloning and functional characterization of novel large conductance calcium-activated potassium channel beta subunits, hKCNMB3 and hKCNMB4. J. Biol. Chem. 275, 6453-6461 (2000). 
4. Wallner, M., Meera, P. \& Toro, L. Molecular basis of fast inactivation in voltage and $\mathrm{Ca}^{2+}$-activated $\mathrm{K}^{+}$channels: a transmembrane beta-subunit homolog. Proc. Natl Acad. Sci. USA 96, 4137-4142 (1999).

5. Xia, X. M., Ding, J. P. \& Lingle, C. J. Molecular basis for the inactivation of $\mathrm{Ca}^{2+}$ - and voltage-dependent BK channels in adrenal chromaffin cells and rat insulinoma tumor cells. J. Neurosci. 19, 5255-5264 (1999).

6. Yan, J. \& Aldrich, R. W. BK potassium channel modulation by leucine-rich repeat-containing proteins. Proc. Natl Acad. Sci. USA 109, 7917-7922 (2012).

7. Barrett, J. N., Magleby, K. L. \& Pallotta, B. S. Properties of single calciumactivated potassium channels in cultured rat muscle. J. Gen. Physiol. 331, 211-230 (1982).

8. Latorre, R., Vergara, C. \& Hidalgo, C. Reconstitution in planar lipid bilayers of a $\mathrm{Ca}^{2+}$-dependent $\mathrm{K}+$ channel from transverse tubule membranes isolated from rabbit skeletal muscle. Proc. Natl Acad. Sci. USA 79, 805-809 (1982).

9. Xia, X. M., Zeng, X. \& Lingle, C. J. Multiple regulatory sites in largeconductance calcium-activated potassium channels. Nature 418, 880-884 (2002).

10. McManus, O. B. et al. Functional role of the beta subunit of high conductance calcium-activated potassium channels. Neuron 14, 645-650 (1995).

11. Yang, C., Zeng, X. H., Zhou, Y., Xia, X. M. \& Lingle, C. J. LRRC52 (leucine-rich-repeat-containing protein 52), a testis-specific auxiliary subunit of the alkalization-activated Slo3 channel. Proc. Natl Acad. Sci. USA 108, 19419-19424 (2011)

12. Xia, X. M., Ding, J. P., Zeng, X. H., Duan, K. L. \& Lingle, C. J. Rectification and rapid activation at low $\mathrm{Ca}^{2+}$ of $\mathrm{Ca}^{2+}$-activated, voltage-dependent $\mathrm{BK}$ currents: consequences of rapid inactivation by a novel beta subunit. J. Neurosci. 20, 4890-4903 (2000).

13. Uebele, V. N. et al. Cloning and functional expression of two families of beta-subunits of the large conductance calcium-activated $\mathrm{K}^{+}$channel. J. Biol. Chem. 275, 23211-23218 (2000).

14. Meera, P., Wallner, M. \& Toro, L. A neuronal beta subunit (KCNMB4) makes the large conductance, voltage- and $\mathrm{Ca}^{2+}$-activated $\mathrm{K}^{+}$channel resistant to charybdotoxin and iberiotoxin. Proc. Natl Acad. Sci. USA 97, 5562-5567 (2000).

15. Knaus, H. G., Eberhart, A., Kaczorowski, G. J. \& Garcia, M. L. Covalent attachment of charybdotoxin to the beta-subunit of the high conductance $\mathrm{Ca}(2+)$-activated $\mathrm{K}+$ channel. Identification of the site of incorporation and implications for channel topology. J. Biol. Chem. 269, 23336-23341 (1994).

16. Dolan, J. et al. The extracellular leucine-rich repeat superfamily; a comparative survey and analysis of evolutionary relationships and expression patterns. BMC Genomics 8, 320-343 (2007).

17. Liu, G. et al. Location of modulatory beta subunits in BK potassium channels. J. Gen. Physiol. 135, 449-459 (2010).

18. Wu, R. S. et al. Location of the beta 4 transmembrane helices in the BK potassium channel. J. Neurosci. 29, 8321-8328 (2009).

19. Liu, G. et al. Locations of the betal transmembrane helices in the BK potassium channel. Proc. Natl Acad. Sci. USA 105, 10727-10732 (2008).

20. Wang, Y. W., Ding, J. P., Xia, X. M. \& Lingle, C. J. Consequences of the stoichiometry of Slo1 alpha and auxiliary beta subunits on functional properties of large-conductance $\mathrm{Ca}^{2+}$-activated $\mathrm{K}^{+}$channels. J. Neurosci. 22, 1550-1561 (2002).
21. Gonzalez-Perez, V., Xia, X. M. \& Lingle, C. J. Functional regulation of BK potassium channels by gamma1 auxiliary subunits. Proc. Natl Acad. Sci. USA 111, 4868-4873 (2014).

22. Orio, P. \& Latorre, R. Differential effects of beta 1 and beta 2 subunits on BK channel activity. J. Gen. Physiol. 125, 395-411 (2005).

23. Xia, X. M., Ding, J. P. \& Lingle, C. J. Inactivation of BK channels by the $\mathrm{NH} 2$ terminus of the beta2 auxiliary subunit: an essential role of a terminal peptide segment of three hydrophobic residues. J. Gen. Physiol. 121, 125-148 (2003).

24. Hoshi, T., Pantazis, A. \& Olcese, R. Transduction of voltage and $\mathrm{Ca}^{2+}$ signals by Slo1 BK channels. Physiology (Bethesda) 28, 172-189 (2013).

25. Sun, X. et al. The interface between membrane-spanning and cytosolic domains in $\mathrm{Ca}(2)+$-dependent $\mathrm{K}+$ channels is involved in beta subunit modulation of gating. J. Neurosci. 33, 11253-11261 (2013).

26. Contreras, G. F., Neely, A., Alvarez, O., Gonzalez, C. \& Latorre, R. Modulation of BK channel voltage gating by different auxiliary beta subunits. Proc. Natl Acad. Sci. USA 109, 18991-18996 (2012).

27. Martinez-Espinosa, P. L., Yang, C., Gonzalez-Perez, V., Xia, X. M. \& Lingle, C. $\mathrm{J}$. Knockout of the BK beta2 subunit abolishes inactivation of BK currents in mouse adrenal chromaffin cells and results in slow-wave burst activity. J. Gen Physiol. 144, 275-295 (2014).

28. Zhang, Z., Zhou, Y., Ding, J. P., Xia, X. M. \& Lingle, C. J. A limited access compartment between the pore domain and cytosolic domain of the BK channel. J. Neurosci. 26, 11833-11843 (2006).

\section{Acknowledgements}

This work was supported by GM-081748 to CJL.

\section{Author contributions}

V.G.-P. and C.J.L. designed research, analysed data and prepared the manuscript. V.G.-P collected the data. X.-M.X. contributed new reagents/analytical tools.

\section{Additional information}

Supplementary Information accompanies this paper at http://www.nature.com/ naturecommunications

Competing financial interests: The authors declare no competing financial interests.

Reprints and permission information is available online at http://npg.nature.com/ reprintsandpermissions/

How to cite this article: Gonzalez-Perez, V. et al. Two classes of regulatory subunits coassemble in the same BK channel and independently regulate gating. Nat. Commun. 6:8341 doi: 10.1038/ncomms9341 (2015).

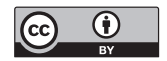

This work is licensed under a Creative Commons Attribution 4.0 International License. The images or other third party material in this article are included in the article's Creative Commons license, unless indicated otherwise in the credit line; if the material is not included under the Creative Commons license, users will need to obtain permission from the license holder to reproduce the material. To view a copy of this license, visit http://creativecommons.org/licenses/by/4.0/ 